\title{
Journal Reviews and Revisions: Advice from an Early Career Panel Discussion
}

\author{
Paul Chan ${ }^{1}$, Carrie Dossick ${ }^{2}$, Miriam Hacker ${ }^{3}$, Timo Hartmann ${ }^{4}$, \\ Amy Javernick-Will ${ }^{5}$, Ashwin Mahalingam ${ }^{6}$, and Vedran Zerjav ${ }^{7}$
}

\footnotetext{
${ }^{1}$ Faculty of Architecture and the Built Environment, Delft University of Technology, Julianalaan 134, 2628 BL, Email: P.W.C.Chan@tudelft.nl, Delft, The Netherlands

${ }^{2}$ P.D. Koon Professor of Construction Management, Associate Dean of Research, College of Built Environments, University of Washington, Architecture Hall 130g, Box 351610, Email: cdossick@uw.edu, Seattle, WA 98195, United States

${ }^{3}$ Postdoctoral Researcher, Environmental Social Sciences Department, Eawag, Swiss Federal Institute of Aquatic Science and Technology, Überlandstrasse 133, Email: hackermiriam@gmail.com, 8600 Dübendorf, Switzerland ${ }^{4}$ Professor, Civil Systems Engineering Department, Technical University Berlin, Email: Email: timo.hartmann@tuberlin.de, Berlin, Germany

${ }^{5}$ Associate Professor and Nicholas R. and Nancy D. Petry Professor, Department of Civil, Environmental, and Architectural Engineering, University of Colorado Boulder, Email: amy.javernick@colorado.edu, Colorado Boulder, United States

${ }^{6}$ Associate Professor, Department of Civil Engineering, Indian Institute of Technology Madras, Email: mash@iitm. ac.in, Chennai, India,

${ }^{7}$ Lecturer, School of Construction and Project Management, Bartlett Faculty of the Built Environment, University College London, United Kingdom. Email: v.zerjav@ucl.ac.uk, London, United Kingdom
} 


\section{Introduction}

Peer review is a cornerstone of high-quality research. While attending $\mathrm{PhD}$ programmes, we mostly interact with advisors, however the academic quality of our work is ultimately judged by a broader range of academic peers. For early career researchers, transitioning into independent thought-leaders requires increasing exposure with our community of peers, and inevitably engaging with review practices - both as authors and reviewers. Whilst many $\mathrm{PhD}$ programmes around the world offer training on paper and grant reviews, journal paper reviews remain somewhat vague to many researchers at all levels who haven't had extensive exposure to advisors, editors, and peers who share their understanding of expectations and best practices. This article provides a discussion of the review and revision process for journal articles, including a check-list for each section.

The review process can feel like a 'black box'; authors submit manuscripts with a great deal of uncertainty as to whether the article will be accepted and unsure about the type of comments they will receive from reviewers. As researchers peerreview others' work they may ask themselves: how can I best review and help maintain the integrity of the review process? These challenges motivated us to organize an Engineering Project Organization Society (EPOS) Early Career Forum Panel on writing reviews and submitting manuscripts. Our intention was to provide early career academics with useful advice and discuss aspects that editors, such as those on our panel, care about.

We approached a number of EPOS senior academics with a request to contribute to a friendly informal discussion for early career scholars. These panelists are active in various editorial roles in journals in construction, engineering, and project organization, where early career EPOS scholars are likely to publish their work. Panelists include the following individuals (in alphabetic order and with only editorial affiliations mentioned). Paul Chan (Construction Management and Economics), Timo Hartmann (Engineering, Construction and Architectural Management and ASCE Journal of Construction Engineering and Management), Amy Javernick-Will (Construction Management and
Economics), and Ashwin Mahalingam (Engineering Project Organisation Journal).

While editors make a judgement based on the recommendations of reviewers, it is worth noting that the role of an editor varies depending on the journal being discussed. Some editors play the role of an honest broker, making a judgement based on what the reviewers recommend (this tends to be the majority). However, other editors play the role of an intermediary, often orchestrating a dialogue between authors and reviewers, and providing an editorial steer where there is a lack of clarity or agreement between the reviewers. The editorial steer is also important for facilitating a connection between the manuscript and current conversations in the journal. Editors thus play an important role as gatekeeper of what is acceptable for that particular domain. While our intention was to compose a panel of senior academics with editorial roles in established journals, it should be mentioned that rather than representing the official editorial position and policies of their respective journals, our panelists shared their personal advice and views as experienced authors and reviewers with the early career EPOS group.

We wrote this article as a collection of best practices that were suggested in the panel discussion with checklists to guide researchers in the reviewing process. It is also important to note that this guidance is generalized; each journal has a unique scope and editorial process. We strongly encourage authors and reviewers to interact directly with their editors about the processes we discuss here.

\section{Key Points in the Publication Process}

We structure this note along the timeline of the publication process for a manuscript (Figure 1). This features the role of the author, the reviewers, and editor. Based on this general procedure, we have chosen to focus on the following key points in the process:

1. Developing a manuscript and selecting a journal (author mode)

2. Reading the paper and submitting comments (reviewer mode) 


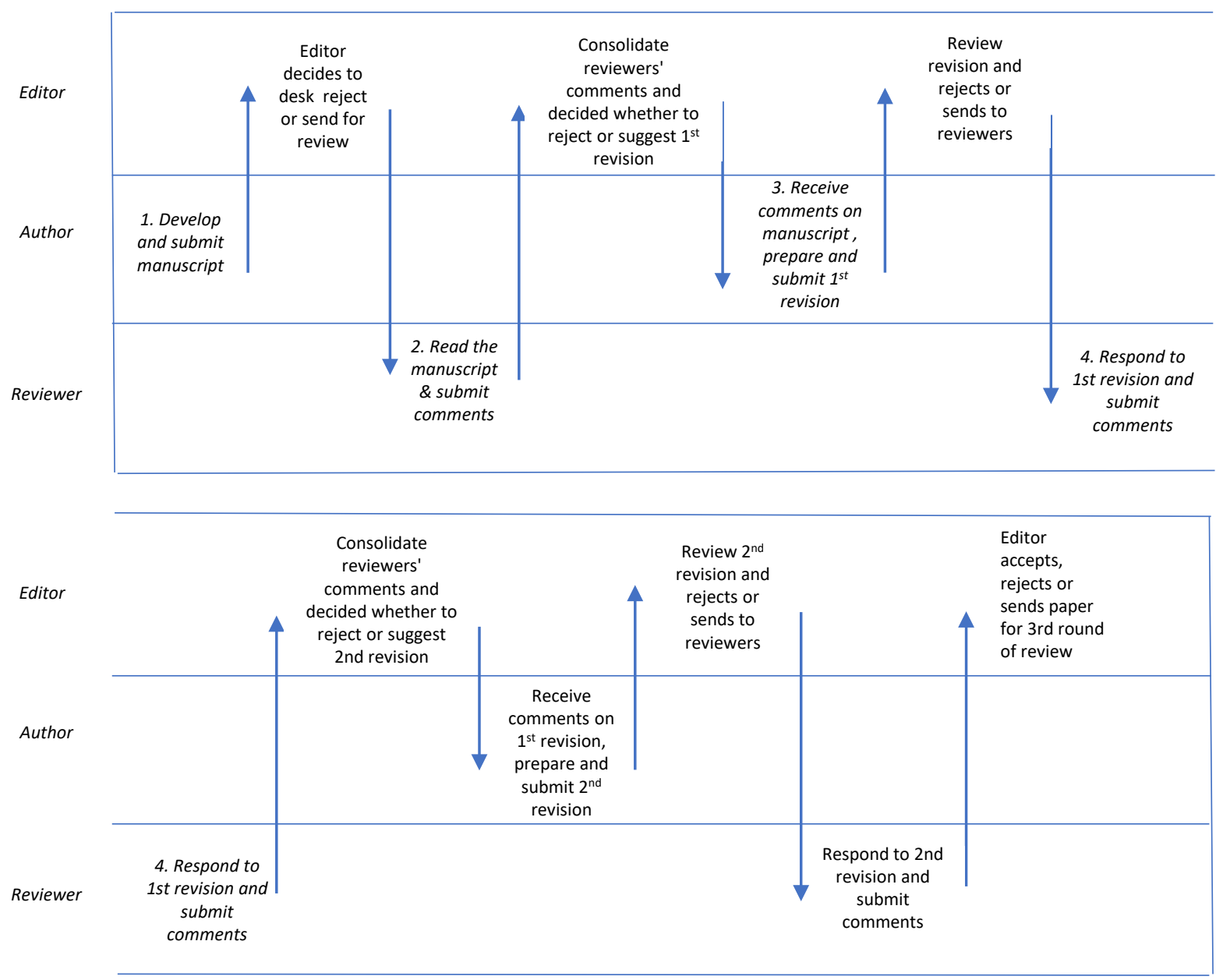

Figure 1 Summary of the general publication process; key points discussed in this article are italicized in blue.

3. Receiving reviewer comments and revising the manuscript (author mode)

4. Responding to author revisions (reviewer mode)

As you read this paper, keep in mind three overarching issues. First, when asked to review a paper, we are faced with the task of understanding the background and main idea of the manuscript without being exposed to the long research process that has underpinned the work. While we do not have direct insight into the research, the manuscript acts as a proxy for what was done. Moreover, we should act as a friendly and constructively critical reviewer while ensuring that the paper contributes to the body of knowledge in the subject area. There are many ways to understand what a contribution to a field of research is, given the diversity of the journals, understanding the nuance between the conversations happening in the journal you are reviewing for will be the best way of unpacking the potential contribution of a manuscript. This usually requires a long engagement with material and contributions published in a journal; it is generally good practice for early career researchers to look up most-cited items and most-read articles in the given journal from the last two or three years to acquire a sense of what the dominant conversations are within that community. We don't suggest that only the most cited contributions in the journal contain the important theoretical and conceptual conversations, but it is a good way to understand whether there is a strong potential for the 
paper to contribute to some of the established conversations in that journal, and to point these out to authors in case they have missed these links. These contributions come either by providing a previously unaddressed perspective on the issue (gap spotting) or revisiting some of the established perspectives (problematising) or suggesting an entirely new domain for study (Shapira, 2011).

Second, as an author, the ability to claim a unique contribution to the journal is the single most important criterion for publication. Traditionally, management and organizationstudies based contributions are expected to be theoretical (as in suggesting new ways for understanding complex phenomena), while contributions to engineering are traditionally more methods/design science based (as in suggesting a method that can be replicated to achieve desired outcomes in practice). This brings us to the first challenge that we face as authors and reviewers. Interdisciplinary fields such as EPO are particularly challenging as the manuscript can be cutting across theoretical conversations in multiple fields (e.g. policy, engineering and social science), which develop separately in their own domains without talking to each other (Davies et al., 2018). As a result, finding a convincing argument for a cross-disciplinary contribution remains a challenge. Besides identifying the contribution to the field, the other challenge in identifying the contribution is that criteria evolve with time. Therefore, the timeline is an important factor in understanding the current contributions in the journal as papers that were published even as recently as 10 years ago can vary substantially in their approach, methodology and structure, to what the same journals consider appropriate today continuously challenging our assumptions about the field.

Third, just as authors look to justify their rationale for selecting a journal for their manuscript, reviewers are similarly assessing whether this same paper will provide a meaningful contribution to the specific journal they are reviewing for. This is just one example of the varying perspectives on similar topics surrounding the peer-review process. Using contributions from the EPOS Early Career Forum Panel, we next provide some of the main signposts to navigate this process, covering both the perspective of authors and reviewers.

\section{Developing a manuscript and selecting a journal (author mode)}

Alright, so you worked hard on the framing of your work, engaged with all the right key literature at the outset, developed a bullet-proof research design and worked hard to achieve a rigorous data collection and analysis. Now, you must decide when you're ready to submit. There are different factors that will impact your decision, such as the length of the review process. Different journals' first round of reviews can range from between two months to over six months; you'll want to consider whether your work is far enough along to be willing to wait for feedback. If you haven't already done so, it's always recommended that you reach out to your direct community (e.g. advisors, colleagues, writing groups) to help with providing initial feedback. Conferences within your community offer wonderful platforms for this. Journals are looking to build off existing conversations in their domain of knowledge through novel and distinct contributions. It is always recommended to choose quality over quantity; editors and reviewers can tell if this paper is only slightly different from other recent publications and you will risk being declined for a lack of original content. In the paper itself, make sure that your approach is clearly stated and easy to follow by readers outside of your research group. One example provided by the panel was to avoid using broad methodological terms (e.g. "grounded theory" - Suddaby (2006)) without detailing the specific steps you took. In other words, you are much better off saying what you did and justifying why you did it in the context of the research question. It should be clear to the reader your rationale for the context and approach (Javernick-Will, 2018); if this is still not clear then reconsider submitting.

Once you've decided that your work is ready, select a good journal to submit your work. Finding the appropriate journal to showcase your work is an important challenge of the publication 
process that every author faces, whether novice or seasoned. The first challenge here is the decision - where do I send my paper? Journals vary in terms of reputation, types of submissions, length of review process, and acceptance rates; all of these factors are a point of consideration for authors. There are two approaches one can take - start developing a paper having a specific journal in mind or have a group of journals in mind with a preference ranking and start engaging with outlets at the top of this list and work your way downwards. There is no one "correct" way to do this, but it is wise to have a journal publication strategy in mind. Remember: journals need papers and authors in the same way that authors need journals to publish their work in. Early in your career you might have submitted papers based on your advisor's preferences but as an independent academic it is now your responsibility of finding a journal that is a good fit. Consider what expectations your employer has for journal rankings-e.g. some institutions do not recognize journals without an impact factor. In addition, note that you are building off the work that is already being published within that journal's community. Therefore, look at your reference list: are you submitting to a journal whose work is incorporated into your discussion? If not, ask yourself why you are choosing this journal. Another way to determine a quality journal is to look at the editorial board: reputation and representation from different backgrounds and universities matter. Finally, you may simply ask yourself 'is this a journal that peers in my field respect?'

Some journals may or may not require authors to submit manuscripts with a cover letter. Especially if you haven't submitted to this journal before, it is crucial to submit a strong cover letter. Yours should include clearly defined practical contributions and the basis for your choice in submitting to this specific journal. This letter is a guidance document as the editor reads your manuscript and makes an initial decision to accept for review or desk reject. Editors see large quantities of manuscripts; letters which provide a strong basis for why this paper is a good fit with the aims, scope and debates of the journal are likely to stand a better chance of making it into the peer-review process. A strong letter summarizes why the journal should be interested in your paper and is worth the additional effort to stand out from the rest.

\begin{tabular}{l} 
Checklist: Developing a manuscript and selecting \\
a journal (author mode) \\
\hline$\square$ Decide when you're ready to submit (Framing, \\
Method, Data, Contribution). \\
$\square$ Be clear about your rationale for the context and \\
approach. \\
$\square$ Select a good journal to submit your work. \\
$\square$ Submit a strong cover letter that explains your \\
contribution and why this journal.
\end{tabular}

\section{Reading the paper and submitting comments (reviewer mode)}

The second stage in the publication process timeline is for the reviewers to read the paper. One of the most common misconceptions is that reviewing a manuscript is quick and easy. Review is instead a deep engagement with the argument submitted by the author and requires developmental criticism (Müller and Klein, 2018). A good reviewer therefore should engage in the practice of 'productive reading,' critically evaluating the development of the argument with its main theoretical building blocks and implications as well as identifying alternative avenues for discussion. This deep engagement is time-intensive and requires thoughtful consideration before agreeing to serve as a reviewer. Editors will send requests for review; it is suggested to accept these requests based on whether you intend to submit to the journal, or you are an expert in the subject manner or method presented in the submitted manuscript.

Once you have agreed to review the manuscript, it is key to set aside the time necessary for a thorough and reflective review, reading through the paper at least two times. Doing so removes a sense of urgency, which often compromises our ability to fully process what is being presented in the manuscript. Whilst it is tempting to quickly skim through the paper, we should be mindful 
that authors have spent a significant amount of time collecting and analyzing data, synthesizing and discussing the results. It is unrealistic to assume that reviewers (who are not involved with the research presented) will be capable of fully processing its content in one read-through, especially with dense theoretical and empirical material often presented in those contributions. On occasions, reviewers may even set aside time to read the key papers that the authors refer to in the paper, to better understand the contributions that the authors are proposing.

In addition to establishing time for review, it is helpful to understand both your strengths and limitations as a reviewer. Editors have different justifications for selecting the reviewers for each manuscript and it is understood that not everyone will have expertise for each aspect of the manuscript. For example, editors might choose reviewers who either extensively published on the subject matter (possibly identified through the links with work cited in the paper) or have substantial expertise in using the methodology being used in the paper. While sometimes a type of study in a given area naturally lends itself to a particular methodology and research design, we should not forget that there are papers whose primary contribution is in the novel application of a methodology in an established research setting. It is therefore important to acknowledge to the editor where we as reviewers can and very importantly cannot contribute meaningful feedback. Sometimes referred to as "reviewer reflexivity," this can look like a reviewer openly acknowledging their intellectual position with respect to the submitted manuscript and noting bias.

Review comments should provide the author with useful feedback to improve the quality of the submitted manuscript and inform the editor's decision on whether or not this is work that should be published. To be useful, comments should be as detailed as possible and draw on specific examples, (Falkenberg and Soranno, 2018). It is also good practice to distinguish between comments on the value of the contribution to theory, practice, and methodological rigor (macro-level), and more detailed comments on improving the clarity along the development of the argument (micro-level). One of the most important comments that should be included in your review is to assess the contribution to knowledge of the manuscript. How does this analysis contribute to the conversations that have already been taking place in previous issues for this specific journal and subject area? We also shouldn't forget that some of the most impactful contributions were ones that establish groundbreaking pathways for new research in the field, rather than extending established ones.

We should remember the scope of the review: to assess the merit and value of the manuscript that has been submitted in relation to extant knowledge, rather than in relation to the paper that we as reviewers would have written if in the author's shoes. An understanding of the positioning and context of the authors' intentions is necessary for providing constructive comments, acknowledging what has been done well and clearly specifying what needs to be changed and why. Along these lines, a good review is candid and has a clear stance on the condition of the paper. Different journals have different editorial policies and reviewers own a large part of the process by assisting the editor in making a decision about the publication. When the editor receives reviewers' comments, they should contain an explicit indication about the likelihood of the paper to be developed to the desired standard in the given timelines for major/minor revision. This decision is dependent on a few factors, for example, how much time is reasonable to allocate for the necessary amendments. Minor revisions might only take a couple weeks to include additional citations or to more clearly articulate the methodology. However, if it seems as though the author would need to completely restructure the paper or make significant conceptual revisions to provide clarity, it is much better to recommend a 'revise and resubmit' or a 'decline' rather than accepting the paper with major revisions. It is much better to be selective at the outset of the process than being optimistic and suggesting very major changes that cannot possibly be addressed in the revision timelines. One of the main frustrations for authors is having a paper rejected in the second or third round of review, after putting a significant amount of effort to address the specific recommendations of 
the set of reviewers allocated to the manuscript on this instance. Engaging more critically with the manuscript at the early stages and rejecting the paper early is much better than taking the authors on a journey with the final destination that is unattainable. Ideally, editors want to avoid excessive iterations on a manuscript. If the paper is not ready, a decline is more appropriate so the authors can do significant revisions before submitting again. Being clear will help respect the time and energy of all parties involved and will help the editor proceed with an informed decision. Finally, while the comments must be critical, it is also good practice to word these criticisms constructively and also to point out the aspects of the paper that have merit, even if the recommendation is to decline the paper. Use the section to provide comments to the editors to clearly indicate your views on the paper and whether the editor should or should take it through the review process.

\section{Checklist: Reading the paper and submitting} comments (reviewer mode)

\section{Reading the paper}

$\square$ Set aside time for a thorough review.

$\square$ Read through the manuscript at least two times.

$\square$ Understand and clearly communicate your

strengths and limitations for the topic presented in

the manuscript.

\section{Submitting comments}

$\square$ Assess the academic contribution.

$\square$ Remember the scope of your review.

$\square$ Provide constructive comments, acknowledging what has been done well.

$\square$ Be candid and have a clear stance on the condition of the paper.

\section{Receiving reviewer comments and revising the manuscript (author mode)}

Receiving peer reviews can be an emotional process where you feel personally attacked and discouraged in your work. These feelings are real; however, the EPOS Early Career Forum panelists recommend reframing how you look at reviewer comments. Take reviewer comments seriously, but not personally. Reviewers spend time trying to understand your work, where you are coming from and understand the contributions you seek to make. Rather than considering their feedback as an attack on your work, their comments are an opportunity to strengthen your paper by highlighting the areas where more clarification is needed. It is not a value judgement on the work itself. Rest in the assurance that all research endeavors are valuable. Every peer review, even the grumpy reviewer, provides you an opportunity to see how others understand your work and what requires clarity. Only you understand the full picture, and in a summative article like a journal paper, things are left unsaid. The craft of writing journal papers is framing and articulating the position you are taking and the contribution you are making, explaining the research methodology, rationale, and presenting the data. Reviewers' comments provide you feedback on what they were able to understand, what was left out in explaining the research, and what contribution they see you making to the journal. If the reviewers' comments indicate that they are not seeing the points made as you intended, then this should provide you with the cues for clarifying your text.

Of course, the reality is that initial receipt of comments can be difficult. If they are too frustrating, allow yourself time to digest and address comments properly. Put them in a drawer and revisit them when you can reflect on and address the comments with a more analytic and constructive frame of mind. In the end, they will make your writing stronger and improve your paper, even if you do not agree with every comment. It is better to have this feedback during the review process rather than with the broader academic community once an article is published. Once you have taken the time to absorb the reviewer comments, map out what it will take to address comments. Do you consider the comments to require minor or major changes? If major, map out what changes are necessary and give yourself a sufficient amount of time to adequately address comments. If minor, work to turn around the paper quickly to reduce time to publication or re-review, when reviewers still recall the paper and their suggestions. 
Just as you have put time and energy into writing your manuscript, it is beneficial to assume that the reviewer has taken time to provide comments for this work. As such, be systematic with your response to reviewer comments. In your response letter, respond to each comment by each reviewer and show, as applicable, what changed as a result. Do not just say that changes have been made or comments have been addressed. $\mathrm{Be}$ as specific as possible to explain how, in what ways and where in the revised manuscript. Depending on the number of comments, it could be a bullet point format in the response letter, or a table. The more comments, the clearer you need to be. While it is necessary to meet the editor's requirements in your revision, be intentional with the revisions you integrate in the manuscript. If reviewers contradict each other, indicate this contradiction along with what changes you elect to make and your rationale for making, or not making, those changes. Avoid compromising the structure of your paper in order to satisfy comments. If a comment is made about the need for a stronger theoretical contribution, this needs to be integrated throughout the paper, not just in the literature review. If you lack integration, you will compromise the flow of a paper, undermining your findings. Stay true to the original intent of the paper unless you are convinced in changing based on a reviewer's comment. If you disagree with a comment by a reviewer, respond logically as to why you think the comment did not need to be addressed in the manuscript.

Be respectful of the time that has been taken to provide feedback and respond appropriately. When making revisions, utilize your limitations section, not only to explain the constraints of your analysis, but also the possibilities for future work. Some of the reviewer comments about why you did or did not include certain variables in your analysis are a great point of discussion for future work. The same holds for general comments to methodological issues, such as criticism to how data was collected. This is why journals and the academic community exist: to build off of each other's work and continue moving forward in better understanding the world around us!

\begin{tabular}{|l|}
\hline $\begin{array}{l}\text { Checklist: Receiving reviewer comments and } \\
\text { revising the manuscript (author mode) }\end{array}$ \\
\hline Receiving reviewer comments \\
$\square$ Take reviewer comments seriously, but not \\
personally. \\
$\square$ Allow yourself time to digest and address \\
comments properly. \\
$\square$ Map out what it will take to address comments. \\
Revising the manuscript \\
$\square$ Be systematic with your response to reviewer \\
comments. \\
$\square$ Be intentional with the revisions you integrate in \\
the manuscript. \\
$\square$ Utilize your limitations section. \\
\hline
\end{tabular}

\section{Responding to author revisions (reviewer mode)}

The next step in the publication journey is receiving the authors' response to the reviewers' comments. Just like we recommended for authors, it is critical as a reviewer to not take disagreement personally. Hopefully the author has addressed review comments thoroughly; read through these changes and author comments and give yourself time to process them. It's never a good idea to respond immediately, especially if there is disagreement between yourself and the author. Limit additional comments to within the scope of your original review. This avoids keeping the author feeling like they are working with a moving target and underlines the importance of providing a thorough initial review. If the manuscript still requires significant work, be realistic with the number of iterations and feel empowered to recommend to the editor that they decline the manuscript if the iterations move beyond the average number of iterations (e.g. two to four iterations, depending on the journal) or if you feel that the author is not adequately addressing comments. This requires transparent communication between you and the editor. As mentioned previously, each journal has its own specific relationship between reviewers and editors and it's always important to clarify this. Several journals have separate sections for providing comments to authors and comments to the editors. The latter is often left blank as 
reviewers may feel that their overall perspective on the paper is evident from their comments to the authors. Often this is not so. Use the section meant to provide comments to the editors to clearly indicate your views on the paper and whether the editor should or should to continue to take it through the review process.

A best practice to consider in the review process is that being critical is only a means to an end - not a purpose in its own right. As academics, we are often trained to identify the shortcomings and weaknesses in the argument, however, we should also acknowledge that an important role of the reviewer is to appreciate all the effort that went into the development of the paper. Authors have different backgrounds, supervisory experiences and publication pressures, yet we should remember that they all share a genuine passion and curiosity to learn and advance knowledge. Therefore, the argument in the review process should be a balanced and developmental one, acknowledging both the merits and the potential for improvement.

\section{Checklist: Responding to author revisions} (reviewer mode)

$\square$ Do not take disagreement personally.

$\square$ Limit additional comments to within the scope of your original review.

$\square$ Be realistic with the number of iterations.

\section{Final comments}

Figure 1 provides an overview of the general publication process. The peer-review process for academic publishing is an opportunity for strengthening the integrity for conversations within the academic community. The EPOS Early Career Forum Panel shared their insights and advice for the reviewing and submitting process with the hopes of addressing major challenges or questions faced by individuals engaging with this process. Thoughtful consideration, respect, productive feedback, and time are all key characteristics. Opinions were shared on behalf of a diverse range of panelists and editorial members. At the Engineering Project Organization Journal (EPOJ), interdisciplinary work is promoted that addresses challenges that project organizations face. We look forward to having you be a part of EPOJ as an author, a reviewer and as a reader. The checklists presented in this article offer guidance to the kinds of practices we would like authors and reviewers to follow to ensure that we engage in high-quality debates on scholarship and co-create articles that can make an impact on the project organization community - both in theory and in practice.

\section{References}

Davies, A., Manning, S. and Söderlund, J. (2018), "When neighboring disciplines fail to learn from each other: the case of innovation and project management research", Research Policy, Vol. 47 No. 5, pp. No. 965-979. 10.1016/j. respol.2018.03.002

Falkenberg, L.J. and Soranno, P.A. (2018), "Reviewing reviews: an evaluation of peer reviews of Journal article submissions", Limnology and Oceanography Bulletin, Vol. 27 No. 1, pp. 1-5. 10.1002/lob.10217

Javernick-Will, A. (2018), "Rationale: the necessary ingredient for contributions to theory and practice", Construction Management and Economics, Vol. 36 No. 8, pp. 423-424. 10.1080/01446193.2018.1487910

Müller, R. and Klein, G. (2018), What Constitutes a Contemporary Contribution to Project Management Journal ${ }^{\circledR}$ ? Los Angeles, CA, SAGE Publications Sage CA.

Shapira, Z. (2011), "“I've Got a Theory Paper-Do You?": Conceptual, Empirical, and Theoretical Contributions to Knowledge in the Organizational Sciences", Organization Science, Vol. 22 No. 5, pp. 1312-1321. 10.1287/orsc.1100.0636

Suddaby, R. (2006), "From the editors: what Grounded theory is not", Academy of Management Journal, Vol. 49 No. 4, pp. 633-642. 10.5465/ amj.2006.22083020 